\title{
Eficácia da gestão de pontes rodoviárias à luz das práticas de inspeção brasileiras
}

\author{
Caroline Buratto de Lima e Oliveira ${ }^{1}$, Marcelo Greco ${ }^{2}$, Camila Esteves Romeiro ${ }^{3}$, \\ Juliane Venturelli Silva Lima ${ }^{4}$, Rafael Arêdes Couto ${ }^{5}$ \\ 1Universidade Federal de Minas Gerais - UFMG, Brasil, carolineburatto@yahoo.com.br \\ 2Universidade Federal de Minas Gerais - UFMG, Brasil, mgreco@dees.ufmg.br \\ 3Universidade Federal de Minas Gerais -UFMG, Brasil, romeiro.camila@gmail.com \\ ${ }^{4}$ Universidade Federal de Minas Gerais - UFMG, Brasil, juliane.venturelli@gmail.com \\ ${ }^{5}$ Universidade Federal de Minas Gerais - UFMG, Brasil, rafaelcouto2111@gmail.com
}

\section{Recebido:}

17 de setembro de 2018

Aceito para publicação:

13 de novembro de 2018

Publicado:

31 de dezembro de 2019

Editor de área:

Francisco Thiago Aragão

\section{Palavras-chaves:}

Gestão de pontes,

Taxas de deterioração de pontes,

Cadeias de Markov.

\section{Keywords:}

Bridge management,

Bridge deterioration rates,

Markov chains.

DOI:10.14295/transportes.v27i4.1840

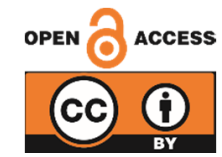

\section{RESUMO}

O controle do estado de condição de cada elemento da infraestrutura de transportes é fundamental para assegurar a operação do sistema a partir de um nível de segurança definido. Para a gestão desses elementos é necessário conhecer as características do parque de obras nacional, seu histórico, estado atual e a previsão do seu estado futuro através de modelos de deterioração - módulo crucial dos sistemas de gestão. Este artigo descreve o atual cenário da gestão de pontes rodoviárias no país e avalia as metodologias de inspeção existentes a partir da aplicação de técnicas de determinação de taxas de deterioração pelo método de cadeias de Markov. Os dados usados pertencem às pontes da malha rodoviária do estado de São Paulo com técnicas de inspeção diversas. Através dos resultados é possível constatar os avanços necessários às práticas existentes no país, considerando suas possíveis implicações sob um gerenciamento eficaz dessas obras.

\begin{abstract}
The management of the asset components of the transportation system infrastructure is crucial to ensure active operation, suitable level of safety and security within budgetary constraints. For this purpose, the full knowledge of the characteristics of road network elements is required, including its history, both current and predicted future conditions through deterioration models - the fundamental module of infrastructure management systems. This article describes the main prediction practices of management of the Brazilian road bridges and evaluates the effectiveness of the existing inspection methodologies to forecast bridge deterioration rates using Markov chain method. Deterioration rates were estimated by using the available data collected in bridges inspections carried out in São Paulo State's roads, based on the most common practices. The presented results required improvements in bridge inspection methods in order to enable improvements to advanced management approaches and increase the quality of the Brazilian entire road network.
\end{abstract}

\section{INTRODUÇÃO}

A gestão da infraestrutura rodoviária busca obter a potencialização das intervenções de Manutenção, Restauração e Reforço (MR\&R) com base em determinado orçamento disponível ou delimitado. A atividade de gerenciar e programar inspeções de pontes com devido registro dos dados para posterior análise e definição de ações de manutenção é conhecida como gestão de pontes ou bridge management (HURT e SCHROCK, 2016).

A limitação orçamentária na gestão de pontes depara-se com os altos custos associados às obras de MR\&R. Para superar este entrave, exige-se um planejamento minucioso com o 
desenvolvimento de abordagens sistemáticas, visando a otimização das ações de MR\&R em pontes através de avançadas ferramentas gerenciais. Essas abordagens devem estar baseadas em análises de desempenho, com foco na redução do custo do ciclo de vida e na extensão da vida útil das estruturas, com níveis de segurança, confiabilidade e manutenção apropriados.

As pontes rodoviárias são sistemas estruturais complexos que se deterioram com o tempo, com taxas diferenciadas de degradação, sujeitas à presença de diversos fatores, tais como: desgaste, fadiga, agentes ambientais agressivos, aumento das cargas de tráfego, programas de manutenção insuficientes, problemas construtivos e projetos inadequados. Além disso, a interação entre esses fatores pode acelerar consideravelmente a taxa de degradação dessas estruturas (MORCOUS, 2000).

0 parque de obras de pontes rodoviárias do Brasil encontra-se envelhecido, com maior parte de suas obras construídas durante a década de 1970 (MENDES, 2003). A condição econômica vivenciada até o final do milênio causou um lapso dos investimentos no setor rodoviário, refletindo na escassez de serviços de manutenção dessas obras (CAMPOS NETO, 2018).

Neste sentido, o Departamento Nacional de Infraestrutura em Transportes (DNIT) conduziu no período de 2013 a 2015 a inventariança e a inspeção de pontes da malha rodoviária federal sob sua administração, utilizando o "Sistema de Gestão de Pontes" - SGO (DNIT 2016) desenvolvido pelo Órgão. Os resultados obtidos classificaram 1.780 obras no estado de "observação" de um total de aproximadamente 6.000 pontes sob a jurisdição da Autarquia, apresentando significativos desafios tecnológicos e econômicos para as próximas décadas.

Um gerenciamento eficaz de pontes da infraestrutura rodoviária depende do conhecimento acurado das condições atuais dessas obras, da qualidade ou da precisão do levantamento dessa condição, da estimativa do seu comportamento deteriorativo ao longo dos anos e, finalmente, dos custos associados aos serviços de intervenção. 0 conhecimento das taxas de deterioração das pontes e, portanto, a previsão de seu comportamento futuro pode fornecer subsídios fundamentais para a tomada de decisão dos gestores de rodovias. 0 objetivo do estudo é verificar a eficácia dos correntes procedimentos de inspeções em pontes brasileiras em promover o conhecimento do comportamento deteriorativo dessas obras, ou ainda, avaliar como essas informações podem contribuir para um gerenciamento eficiente dessas estruturas.

\section{INDICADORES USADOS NO PLANEJAMENTO ESTRATÉGICO DE INTERVENÇÕES EM PONTES}

Os programas de gestão de pontes existentes ou Bridge Management Systems (BMS), internacionais ou nacionais, estão fundamentados nas análises de "Índices de Condição" ou "Nota Técnica" (IC). O IC pode ser atribuído por elemento, por conjunto de elementos ou para a totalidade da obra e reflete o resultado da avaliação do estado de condição daquela construção. 0 IC usualmente contém uma qualificação subjetiva da capacidade portante da ponte, relacionando um valor numérico de acordo com a sua segurança estrutural. Por possuir importante informação, o IC é o principal índice utilizado nas decisões gerenciais para a determinação das ações necessárias de intervenção.

Atualmente no Brasil, existem diversos procedimentos de inspeção de pontes rodoviárias em paralelo a procedimentos próprios desenvolvidos pelas empresas gestoras de trechos rodoviários. Considerando a maior extensão de rodovias sob uma mesma administração, pode-se destacar três normas ou regulamentos distintos com maior utilização no Brasil: a norma NBR 9452/2016 da Associação Brasileira de Normas Técnicas (ABNT, 2016), a norma DNIT PRO 10 
do DNIT (DNIT, 2004) utilizados pelo próprio Órgão e pela Agência Nacional de Infraestrutura em Transportes (ANTT) e a Resolução ETC-0021 da Agência de Transporte do Estado de São Paulo (ARTESP, 2007).

As classificações da condição de pontes pelas normas do DNIT e da ABNT são realizadas em cinco níveis de IC, sendo a pior condição o nível 1 e a melhor condição o nível 5. Nas normas, o IC é atribuído para a condição geral (nota geral) e para cada região da estrutura da ponte dividida em superestrutura, mesoestrutura, infraestrutura e acessos. Apesar da nova versão da norma NBR 9452/2016 incluir a classificação de acordo com os parâmetros de durabilidade e de funcionalidade, as inspeções conduzidas até 2016 contavam apenas uma qualificação geral da estrutura, possuindo maior tendência na atribuição subjetiva da condição estrutural dos seus elementos.

A Resolução ETC-0021 de 2007 da Agência de Transporte do Estado de São Paulo prevê classificações de acordo com os parâmetros estruturais, funcionais e de durabilidade. Para cada parâmetro podem ser atribuídos três estados operacionais $A, B$ e C significando, respectivamente, "Bom", "Regular" e "Ruim. Os estados operacionais são ainda subdivididos em A5, A4, B4, B3, B2, C2, C1, C0, resultando em 8 níveis de classificação, sendo A5 o melhor estado e C0 o pior estado de condição.

Finalmente, algumas empresas ainda adotam procedimentos próprios de inspeção ou de complementação dessas análises existentes. Da análise de relatórios de inspeções rotineiras em pontes elaborados por diversos administradores de rodovias é possível verificar a ausência na padronização do registro das informações, bem como a falta de uniformização dos critérios técnicos adotados (OLIVEIRA e FONSECA, 2013). Na sua grande maioria as análises possuem grande viés qualitativo, com diferentes níveis de detalhamento das observações ou dados. A deficiência na alocação e padronização dos registros pode ser verificada também em outras áreas de pesquisa de infraestrutura rodoviária. Como exemplo, em seu trabalho sobre gerenciamento de pavimentos, Lima (2007) cita diversas restrições na implementação das técnicas de gestão nas rodovias da sua pesquisa decorrentes da ausência, qualificações demasiadamente qualitativas ou mesmo da insuficiência de dados necessários para o desenvolvimento da pesquisa.

\section{MODELOS DE DETERIORAÇÃO EM PONTES}

Os primeiros modelos de deterioração criados no setor de infraestrutura rodoviária foram desenvolvidos para implementação nos sistemas de gestão de pavimentos - Pavement Management Systems (PMS), no início da década de 80 (MORCOUS e HATAMI, 2011). Os processos de deterioração de pavimentos rodoviários se diferenciam dos processos de deterioração de pontes rodoviárias devido aos diferentes tipos de materiais empregados e ao específico comportamento estrutural.

Os modelos de deterioração de pontes podem ser de natureza empírica ou mecanicista de acordo com a natureza dos dados utilizados. Apesar das diferentes abordagens, esses métodos podem ser classificados em modelos de deterioração determinísticos, estocásticos e de inteligência artificial (SETUNGE e HASAN, 2011). A abordagem probabilística é a mais utilizada através dos modelos estocásticos e considera que a deterioração ao longo do tempo é desconhecida, determinando probabilidades para que ela ocorra de acordo com determinada relação ou função. Nesse modelo o IC é tomado como variável aleatória de entrada para a análise (MORCOUS et al., 2002). 
Dentre todas as técnicas de modelos estocásticos, destacam-se as Cadeias de Markov por sua grande utilização para a formulação de modelos de deterioração de pontes (KONG et al., 2010). As Cadeias de Markov utilizam as probabilidades de transição periódicas entre os diferentes níveis de IC, permitindo a previsão da evolução desse parâmetro ao longo do tempo (AASHTO, 2013). 0 método é usado em nos principais sistemas de gestão de pontes da América do Norte e da Europa (GOLABI e SHEPHARD, 1997). A técnica é capaz de mensurar a dependência com o tempo e a incerteza dos processos de deterioração das pontes com fácil implementação computacional e simplicidade de uso (MORCOUS, 2006).

As probabilidades de uma ponte mudar de estado ou alterar o IC são representadas por uma matriz ( $n \times n)$, sendo " $n$ " os diferentes níveis do IC constituindo a denominada Matriz de Transição de Probabilidades - MTP (Transition Probability Matrix) (NORRIS, 1998). A probabilidade de um elemento transitar do estado " $i$ " num tempo inicial para um estado " $j$ " num tempo $\Delta$ t é dada por:

$$
P_{(\Delta t) i j}=P_{r}\left(X_{\Delta t}=j \mid X_{\Delta t}=i\right)
$$

Analogamente, a probabilidade de um elemento permanecer no estado " $i$ " num tempo $\Delta$ t é dada por:

$$
P_{(\Delta t) i i}=P_{r}\left(X_{\Delta t}=i \mid X_{\Delta t}=i\right)
$$

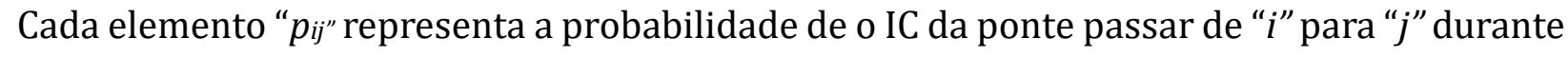
um determinado intervalo de tempo $(\Delta \mathrm{t})$. A estrutura da MTP é descrita pela seguinte matriz:

$$
P=\left[p_{i j}\right]=\left[\begin{array}{cccc}
p_{11} & p_{12} & \ldots & p_{1 n} \\
p_{21} & p_{22} & \ldots & p_{2 n} \\
\vdots & \vdots & \ddots & \vdots \\
p_{m 1} & p_{m 2} & \ldots & p_{m n}
\end{array}\right]
$$

Os dados dos ICs devem seguir um intervalo regular ou equidistante, discreto e inteiro, podendo, por exemplo, ser igual a um ou dois anos (ALMEIDA, 2013).

Os intervalos dos dados irão refletir sobre os resultados probabilísticos, se o intervalo for de um ano, $P_{i j}$ é a probabilidade de uma ponte apresentar determinado IC em 1 ano e $P_{i i}$ de permanecer.

Para pequenos bancos de dados, situação comumente encontrada, dado que as pioneiras pesquisas na área tiveram seu início apenas a partir da década de 90, a probabilidade pode ser calculada seguindo-se a denominada abordagem de frequência (JIANG et al., 2010, SETUNGE e HASAN, 2011, MUÑOZ et al., 2016). Sendo $P_{i j}$ a probabilidade de transição do elemento passar do IC "i" para o IC "j" é estimada por

$$
P_{i j}=\frac{n_{i j}}{n_{i}}
$$

em que $n_{i j}$ é o número de transições do IC "i" para o IC "j" em um período determinado e $n_{i}$ é o total de elementos no estado "i" antes da transição.

\section{MÉTODO DE DETERMINAÇÃO DAS TAXAS DE DETERIORAÇÃO DE PONTES DO ESTADO DE SÃO PAULO}

Para o estudo das taxas de deterioração em pontes rodoviárias foram utilizados os dados das inspeções em pontes pertencentes a trechos rodoviários do estado de São Paulo. 0 estado possui diferentes administrações das suas rodovias que se utilizam dos dois procedimentos de inspeções de pontes mais utilizados no país. Os dados foram fornecidos pelas agências ANTT e ARTESP, conforme Tabela 1: 
Tabela 1 - Relação dos trechos rodoviários das pontes da pesquisa.

\begin{tabular}{|c|c|c|c|}
\hline Rodovia & Descrição/Empresa administradora & Trecho & Agência reguladora \\
\hline BR-116 & $\begin{array}{l}\text { Rodovia Presidente Dutra/ } \\
\text { Concessionária da Rodovia } \\
\text { Presidente Dutra S/A }\end{array}$ & Divisa RJ/SP - município de São Paulo. & ANTT \\
\hline BR-116 & $\begin{array}{l}\text { Rodovia Régis Bittencourt/ } \\
\text { Autopista Régis Bittencourt S/A }\end{array}$ & Município de São Paulo - Divisa SP/PR. & ANTT \\
\hline BR-153 & $\begin{array}{l}\text { Rodovia Transbrasiliana/ } \\
\text { Transbrasiliana Concessionária de Rodovias S/A }\end{array}$ & Divisa MG/SP - Divisa SP/PR & ANTT \\
\hline SP-270 & $\begin{array}{l}\text { Rodovia Raposo Tavares/ } \\
\text { Concessionária Auto Raposo Tavares S/A }\end{array}$ & $\begin{array}{l}\text { km 381+703 (entroncamento SP-327) } \\
\text { - Divisa SP/MS. }\end{array}$ & ARTESP \\
\hline $\begin{array}{l}\text { SP-300 } \\
\text { (Trecho 1) }\end{array}$ & $\begin{array}{l}\text { Rodovia Marechal Rondon/ } \\
\text { Viarondon Concessionária de } \\
\text { Rodovias S/A }\end{array}$ & $\begin{array}{l}\text { km 336+500 (Entroncamento SP- } 225 \\
\text { município de Bauru) - km 667+630. }\end{array}$ & ARTESP \\
\hline $\begin{array}{l}\text { SP-300 } \\
\text { (Trecho 2) }\end{array}$ & $\begin{array}{l}\text { Rodovia Marechal Rondon/ } \\
\text { Concessionária Rodovias } \\
\text { do Tietê S/A }\end{array}$ & $\begin{array}{l}\text { km 158+650 (Tietê) - km 336+500 } \\
\text { (Entroncamento SP-225, } \\
\text { município de Bauru). }\end{array}$ & ARTESP \\
\hline SP-101 & $\begin{array}{l}\text { Rodovia Campinas-Tietê/ } \\
\text { Concessionária Rodovias } \\
\text { do Tietê S/A }\end{array}$ & $\begin{array}{l}\text { km 0+000 (município de Campinas) - } \\
\text { km 71+250 (entroncamento SP-127, } \\
\text { município de Tietê). }\end{array}$ & ARTESP \\
\hline
\end{tabular}

A escolha dos trechos da pesquisa procurou equilibrar a extensão das três rodovias federais concessionadas no Estado de São Paulo com outras três rodovias estaduais. As rodovias Presidente Dutra, Régis Bittencourt e Transbrasiliana possuem extensão no estado de São Paulo de $231 \mathrm{~km}, 292 \mathrm{~km}$ e $321 \mathrm{~km}$, respectivamente, somando 844 quilômetros. As rodovias estaduais Raposo Tavares, Marechal Rondon e Campinas-Tietê possuem 266 km, 326 km e 226 km respectivamente, somando $818 \mathrm{~km}$ de extensão do trecho rodoviário estadual (Figura 1).

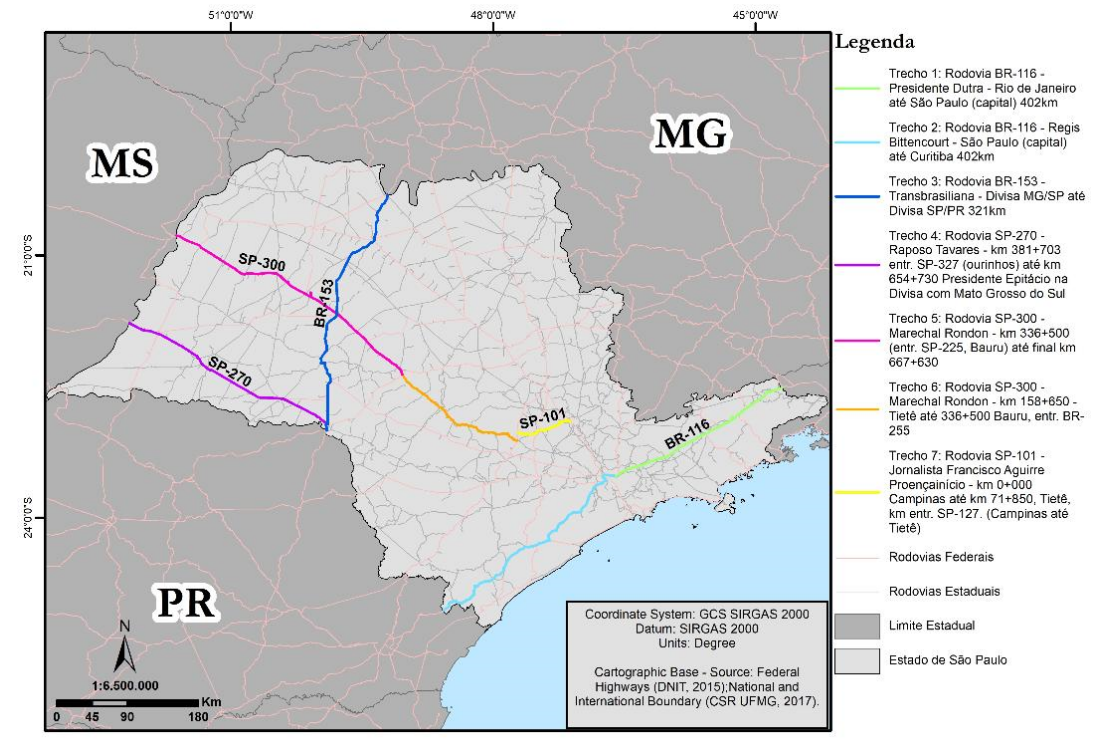

Figura 1. Distribuição das rodovias da pesquisa de deterioração em pontes no estado de São Paulo

Os dados fornecidos pela ANTT são de inspeções rotineiras realizadas de 2009 a 2016 (7 ciclos). Os dados encaminhados pela ARTESP possuem de diferentes períodos, sendo de 2012 a 2016 na SP-270 (4 ciclos) e de 2012 a 2015 na SP-300 do km 336+500 até 667+630 (3 ciclos). No segmento do km 158+650 ao 336+500 da SP-300 e na SP-101 os dados são de 2009 a 2016 (7 ciclos). Os formulários de inspeções foram disponibilizados em formato de texto ou em planilhas eletrônicas ou em documentos digitalizados, somando 907 obras de arte especiais. 
Para o registro dos dados foi desenvolvida uma rotina em VBA (Visual Basic for Applications), organizando as informações para a simulação estocástica. Para a aplicação do método de cadeias de Markov foram desenvolvidas rotinas originais na linguagem de programação $R$ (Project for Statiscal Computing) com interface gráfica no pacote de gráficos ggplot2.

A pesquisa considerou apenas as estruturas de pontes, excluindo-se as passarelas, túneis, bueiros e passagens de gado, devido aos seus distintos comportamentos estruturais. Foram eleitos critérios de filtragem baseados no relatório sobre as taxas de deterioração das pontes do estado de Nova York nos Estados Unidos (AGRAWAL e KAWAGUCHI, 2009) que basicamente sugerem a exclusão dos dados de inspeções com registros incompletos, flutuações pontuais do IC (atribuídas à subjetividade do inspetor) e as quedas abruptas do IC em dois ciclos consecutivos (atribuídas à acidentes ou desastres).

0 período " $t$ " considerado para as avaliações teve sua origem atribuída ao início das inspeções registradas, não sendo considerado como a idade da construção. Em diversos estudos conduzidos na área, a idade da construção é usada como variável " $t$ " de tempo, no entanto seu valor deve ser alterado a cada intervenção que altere significativamente as condições de durabilidade da obra. Como nos dados fornecidos não constavam os registros da data de construção ou das intervenções de melhoria realizadas nessas obras, essa abordagem não pôde ser utilizada (BOLUKBASIL et al., 2004).

Para a aplicação das Cadeias de Markov, as notas atribuídas às pontes estaduais de São Paulo tiveram seus níveis alfabéticos (A, B e C) modificados para uma escala numérica, de 1 a 8 , sendo 1 o IC da pior condição (C0) e 8 o IC da melhor condição (A5). Não foram utilizadas as classificações de funcionalidade e durabilidade por não haver correspondência com as classificações de inspeções das rodovias federais e ainda por não se relacionarem diretamente ao fenômeno de deterioração de interesse da pesquisa. Similarmente, não foram considerados os dados das inspeções cadastrais da rodovia Transbrasiliana porque este era o único trecho do conjunto com essas informações.

Para a análise dos resultados foram elaborados gráficos das curvas de deterioração a partir das probabilidades de transição calculadas na MTP de cada trecho, considerando o início de cada curva em diferentes IC. Além do gráfico, o tempo esperado médio para que cada grupo de pontes atinja determinado IC também foi calculado, visando auxiliar na compreensão das diferentes velocidades de deterioração ao longo dos anos. Como a premissa da utilização das cadeias de Markov considera apenas as pontes que observaram decaimento do IC com queda de apenas um nível no intervalo ( 1 ano), o tempo esperado $E_{Y}\left(T_{X}\right)$ em anos pôde ser obtido através da expressão:

$$
E_{Y}\left(T_{X}\right)=E_{Y}\left(T_{Y-1}\right)=\frac{1}{1-p_{Y Y}}
$$

onde $T_{X}$ é o tempo mínimo, em anos, para que uma ponte atinja, pela primeira vez, o estado de condição X e p $p_{Y Y}$ é a probabilidade que permaneça no estado Y (NORRIS, 1998).

Para a exemplificação da aplicação do método realizou-se a previsão de IC futuro para os grupos de pontes de pesquisa, através da expressão:

$$
E\left(N_{t}\right)=N_{0} \cdot(M P T)^{t}
$$

em que $\mathrm{N}_{0}$ é o número de pontes em determinado IC em determinado ano e $\mathrm{N}_{\mathrm{t}}$ é o número de pontes no IC após t anos.

Finalmente, visando contribuir também para a mensuração da eficácia do método, as MTP usadas na Equação 5 foram elaboradas desconsiderando o último ano do período de inspeções 
de cada grupo de pontes. Ou seja, para uma amostra do período de 2008 a 2016, por exemplo, a nova MTP foi formulada usando apenas os dados de 2008 a 2015. A partir dessa MTP, foi realizada a previsão para o ano de 2016 de acordo com a Equação 5 . 0 valor do desvio também foi calculado e, a partir dos resultados, o número de pontes em cada IC previsto pôde ser comparado com o número de pontes efetivamente classificadas em cada estado no último ano do período.

\section{APRESENTAÇÃO DOS RESULTADOS}

Após o lançamento dos dados e aplicação dos filtros foi possível determinar as MTP de cada grupo de pontes. Os resultados são demonstrados através de matrizes com a indicação dos índices por linha e por coluna para a melhor compreensão das probabilidades calculadas.

\subsection{Rodovias Federais}

Apesar da metodologia de inspeção na Rodovia Presidente Dutra ser específica da empresa administradora, o IC global é informado nas fichas de inspeção. No entanto, os registros dos IC por região da estrutura têm início apenas em 2012, com ausência das notas dos anos de 2013 e 2015, inviabilizando a utilização total dessas informações. As pontes da rodovia no estado de São Paulo somam 81 obras e, após a filtragem, os dados de 71 obras puderam ser usados para o desenvolvimento da MTP de tamanho $5 \times 5$ da Equação 7.

\begin{tabular}{l|lll|l|l|} 
IC & $\mathbf{1}$ & $\mathbf{2}$ & $\mathbf{3}$ & $\mathbf{4}$ & $\mathbf{5}$ \\
$\mathbf{1}$ & 1 & 0 & 0 & 0 & 0 \\
\hline $\mathbf{2}$ & 0,0357 & 0,9643 & 0 & 0 & 0 \\
\hdashline $\mathbf{3}$ & 0 & 0,0655 & 0,9345 & 0 & 0 \\
\hdashline $\mathbf{4}$ & 0 & 0 & 0,0433 & 0,9567 & 0 \\
$\mathbf{5}$ & 0 & 0 & 0 & 0,2500 & 0,75
\end{tabular}

A rodovia Régis Bittencourt possui 177 obras catalogadas no estado de São Paulo. Após a filtragem dos dados, a simulação estocástica pôde ser realizada com os dados de 100 pontes. Dois ciclos com intervalos bastante superiores a 12 meses tiveram os seus dados retirados da amostragem devido à premissa da utilização das Cadeias de Markov, que prevê intervalos regulares. Dessa forma, somente 410 transições das 845 transições existentes de IC puderam ser usadas na montagem da MTP de tamanho 5x5 apresentada na Equação 8:

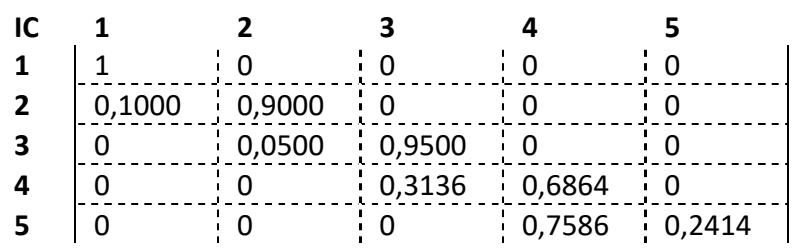

A rodovia Transbrasiliana possui 62 obras catalogadas que após a filtragem dos dados se reduziram a 41 obras aptas para a simulação estocástica. Apesar dos dados disponíveis das inspeções possuírem classificações a partir do $\mathrm{IC}=2$, não existem números de transições do $\mathrm{IC}=2$ para o IC=1 em número suficiente para a determinação da probabilidade de ocorrência dessa mudança. Decorrente desse fato, o resultado é a MTP de tamanho 4X4 da Equação 9:

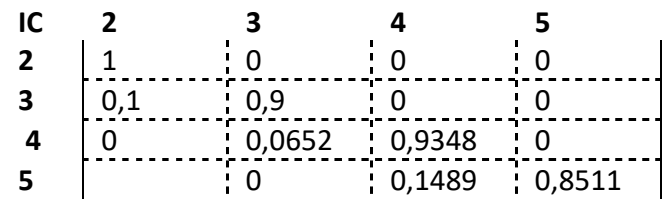


Interpretando a MTP da Equação 7 é possível verificar que uma ponte na rodovia Presidente Dutra com IC=5 tem $24,14 \%$ de probabilidade de mudar seu estado para o IC=4 no próximo ciclo (após 1 ano) e 75,86\% de probabilidade de manter-se no IC=5, observando forte tendência em sair do nível 5 em vista o curto intervalo avaliativo de um ano. Nos níveis IC=4 e IC=3 as probabilidades de mudança para um pior estado são bem menores (6,55\% e 3,57\% respectivamente). De acordo com a MTP das pontes da Rodovia Régis Bittencourt na Equação 8, a probabilidade de uma ponte desse grupo passar do IC=5 para o IC=4 em apenas 1 ano é de 75,86\%. Essa forte taxa de deterioração com início no IC=5 permanece até a estrutura alcançar o IC=3. As pontes da rodovia Transbrasiliana apresentam forte tendência à permanência em todos os estados, destacadamente no IC=4. As maiores tendências de mudança são das transições dos IC extremos, do IC=5 para o IC=4 e do IC=3 para o IC=2.

As probabilidades calculadas nas MTP de cada trecho rodoviário podem ser melhor visualizadas a partir da construção de gráficos de curvas de deterioração, tomando diferentes IC de partida conforme mostra a Figura 2.

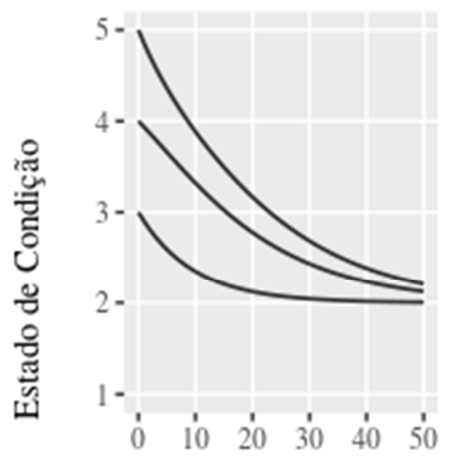

(a)

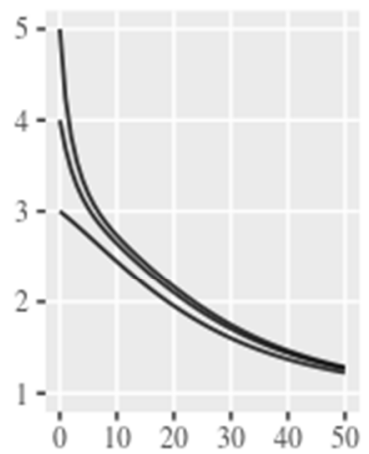

(b)

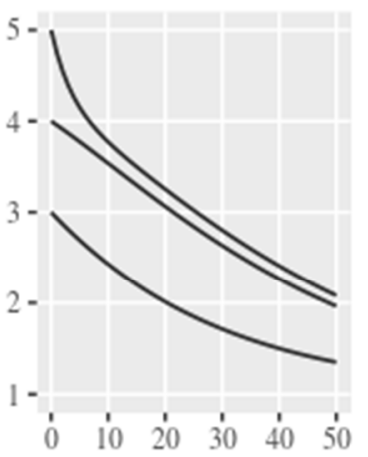

(c)

Tempo - anos

Figura 2. Curvas de Deterioração das Pontes das Rodovias Federais da pesquisa. (a) Rodovia Transbrasiliana (b) Rodovia Régis Bittencourt (c) Rodovia Presidente Dutra

Os gráficos da Figura 2 possuem a característica de suas curvas tangenciarem o mínimo IC da MTP, não atingindo esse valor, pois são construídos a partir dos valores médios previstos. Nos gráficos da Rodovia Transbrasiliana, por exemplo, por não existirem transições do IC=2 para o IC=1, as curvas não atingirão o valor $\mathrm{IC}=2$.

Novas MTP a partir dos dados de IC por partes da estrutura das rodovias federais foram calculadas. No entanto, somente os dados do item "pista de acesso" tiveram volume suficiente para a construção da MTP parcial da Rodovia Presidente Dutra. A partir dessas probabilidades por regiões da estrutura foi possível elaborar os gráficos da Figura 3.

É possível verificar em todos os gráficos da Figura 3 a tendência de maiores taxas de deterioração evidenciados pelas inclinações das curvas para as regiões mais diretamente submetidas ao impacto do tráfego dos veículos, tais como pista de acesso, laje e vigamento principal. Para as pontes da Rodovia Transbrasiliana, a taxa de deterioração dos elementos da pista de acesso possui valor consideravelmente mais elevado, alterando o IC=5 para IC=4 em menos de 5 anos e permanecendo elevada até os primeiros 10 anos. A mesma taxa é bastante diferente para os elementos da mesoestrutura, alterando seu IC de 5 para 4 com o dobro desse tempo, aproximadamente 10 anos. 


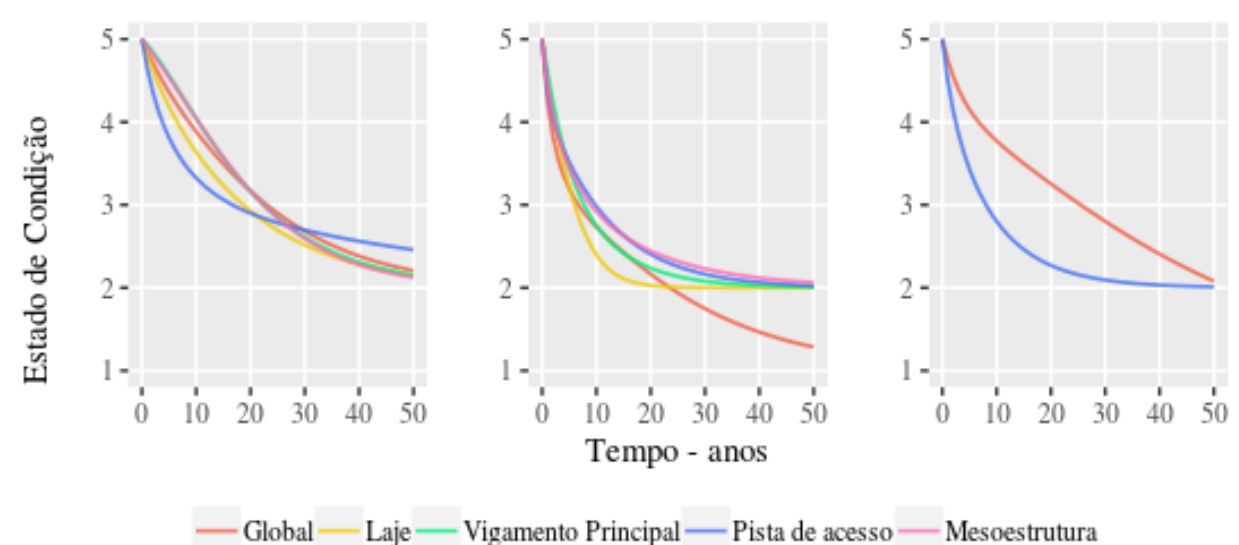

(a)

(b)

(c)

Figura 3. Curvas de deterioração por região das pontes. (a) Rodovia Transbrasiliana (b) Rodovia Régis Bittencourt (c) Rodovia Presidente Dutra

É possível calcular, a partir das probabilidades da MTP de cada trecho, o tempo médio esperado em anos das pontes de cada grupo passarem de um nível Y para Y-1, de acordo com a Equação 4. Os resultados são apresentados na Tabela 2.

Tabela 2 - Tempo médio estimado para mudança de estado das pontes das Rodovias Federais

\begin{tabular}{llll}
\hline \multicolumn{4}{c}{ Período de Transição para um IC inferior (em anos) } \\
\hline Trecho Rodoviário & $5 » 4$ & $4 » 3$ & $3 » 2$ \\
Nova Dutra BR-116 (SP) & 4,0 & 23,1 & 28,0 \\
Régis Bittencourt BR-116 (SP) & 1,3 & 3,2 & 20 \\
Transbrasiliana BR-153 & 6,7 & 15,3 & 10,0 \\
\hline
\end{tabular}

Analisando as rodovias federais, a BR-153 apresentou a menor taxa de deterioração das suas pontes, provavelmente devido ao volume de tráfego médio bastante inferior à BR-116. 0 trecho da Régis Bittencourt apresentou maior taxa de deterioração, bastante superior às demais. Avaliando o banco de dados é possível verificar que das 118 obras do trecho paulista, 43 pontes receberam em algum ano do período a classificação do IC=5. Das 43 pontes com o IC máximo, 41 pontes tiveram queda no IC durante o período, sendo que 27 dessas pontes alteraram para o IC=4 após apenas 1 ano, no ciclo avaliativo seguinte. A taxa permanece alta para os IC mais inferiores, levando a uma ponte mudar do IC=4 para o IC=3 em apenas 3,2 anos.

Finalmente, foram construídas novas MTP desconsiderando-se o último ano do ciclo de cada grupo de pontes. A partir das probabilidades de transição encontradas, foram realizadas estimativas do número de pontes em cada IC para $t=1$, ou seja, para o ano seguinte, usando a Equação 5. Os resultados estão apresentados na Tabela 3, juntamente com desvio associado à previsão.

Apesar dos períodos das inspeções disponíveis das pontes serem relativamente curtos, somando 5 ciclos após retirar o último ano, a previsão do IC para as pontes da Presidente Dutra e Transbrasiliana para o próximo ano possui boa aproximação com os resultados reais com os dados reais variando dentro do intervalo do desvio máximo calculado. A previsão para as pontes da Régis Bittencourt apresenta maior diferença dos resultados e desvios relativos altos devido ao pequeno aproveitamento dos dados fornecidos, dada a restrição do método que prevê a utilização de intervalos regulares para sua aplicação. 
Tabela 3 - Previsão de número pontes das rodovias federais por IC após 1 ciclo

\begin{tabular}{|c|c|c|c|c|c|c|}
\hline \multirow[t]{2}{*}{ Rodovia } & \multirow[t]{2}{*}{ Ano } & \multicolumn{5}{|c|}{$\begin{array}{l}\text { Número de pontes em cada Estado de } \\
\text { Condição }\end{array}$} \\
\hline & & 1 & 2 & 3 & 4 & 5 \\
\hline \multirow{3}{*}{$\begin{array}{l}\text { BR-116 } \\
\text { Régis Bittencourt }\end{array}$} & 2015 (dados) & 0 & 1 & 30 & 62 & 7 \\
\hline & 2016 (dados) & 1 & 0 & 40 & 57 & 2 \\
\hline & Previsão 2016 & $\begin{array}{c}0 \\
(0,30)\end{array}$ & $\begin{array}{c}2 \\
(1,23)\end{array}$ & $\begin{array}{c}48 \\
(3,84)\end{array}$ & $\begin{array}{c}48 \\
(3,82)\end{array}$ & $\begin{array}{c}2 \\
(1,13)\end{array}$ \\
\hline \multirow{3}{*}{$\begin{array}{l}\text { BR-116 } \\
\text { Presidente Dutra }\end{array}$} & 2015 (dados) & 1 & 4 & 21 & 40 & 5 \\
\hline & 2016 (dados) & 1 & 12 & 24 & 52 & 4 \\
\hline & Previsão 2016 & $\begin{array}{c}1 \\
(0,64)\end{array}$ & $\begin{array}{c}13 \\
(1,35) \\
\end{array}$ & $\begin{array}{c}24 \\
(1,90) \\
\end{array}$ & $\begin{array}{c}52 \\
(1,72) \\
\end{array}$ & $\begin{array}{c}3 \\
(0,87) \\
\end{array}$ \\
\hline \multirow{3}{*}{$\begin{array}{l}\text { BR-153 } \\
\text { Transbrasiliana }\end{array}$} & 2015 (dados) & 0 & 0 & 3 & 9 & 17 \\
\hline & 2016 (dados) & 0 & 0 & 3 & 11 & 15 \\
\hline & Previsão 2016 & $\begin{array}{l}0 \\
(-)\end{array}$ & $\begin{array}{c}0 \\
(0,52)\end{array}$ & $\begin{array}{c}3 \\
(0,90)\end{array}$ & $\begin{array}{c}11 \\
(1,64)\end{array}$ & $\begin{array}{c}15 \\
(1,47)\end{array}$ \\
\hline
\end{tabular}

Importante observar que a taxa de deterioração é inversamente proporcional ao tempo estimado para a transição de um estado de condição para o outro, assim, quanto maior a taxa, menor será o tempo necessário para a ponte alcançar um IC inferior de classificação.

\subsection{Rodovias Estaduais}

0 trecho da Raposo Tavares (SP-270) possui 161 obras de arte especiais, sendo 72 transposições de passagens de gado e galerias. Após a filtragem, os dados de 85 pontes foram utilizados. Apesar de existirem pontes classificadas do IC $=2$ ao IC=8, apenas as probabilidades de decréscimo até o IC $=5$ puderam ser calculadas. Isto se deve ao fato de que os IC inferiores somente foram atribuídos no primeiro ano (2012) tendo essas pontes melhorado sua avaliação ou IC no ano seguinte (2013). A inexistência de dados de transição do IC=5 para o IC=4 desse trecho pode encontrar possível explicação nas intervenções de melhoria realizadas ao longo do período avaliativo. 0 menor IC encontrado nos dados é o IC=5, nível bastante distante no menor valor da escala de classificação utilizada (IC=1). Tal fato sugere a provável existência de um nível de desempenho mínimo adotado pela administradora desse trecho rodoviário. A MTP resultante possui tamanho $4 \times 4$ e é apresentada na Equação 10:

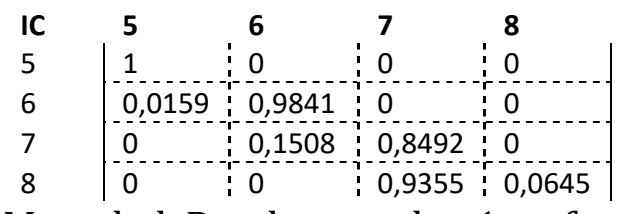

Os dados de vistorias da Marechal Rondon trecho 1, referente do km 336+500 ao km 667+630 somam 307 obras que após a filtragem resultaram em 193 pontes para o cálculo da MTP. Existem classificações de pontes nos níveis $\mathrm{IC}=2$ ao $\mathrm{IC}=8$. No entanto, o número de transições do IC=3 para IC=2 não tiveram quantidade suficiente para se aferir a probabilidade da sua ocorrência, resultando a seguinte MTP de tamanho 6x6 da Equação 11:

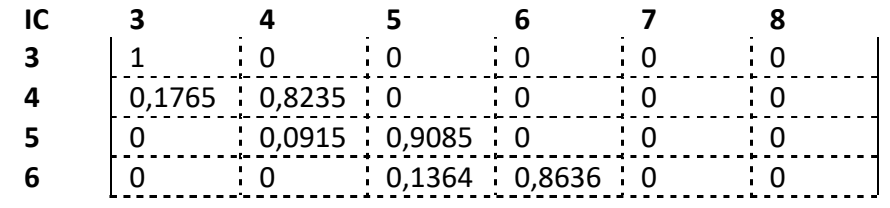




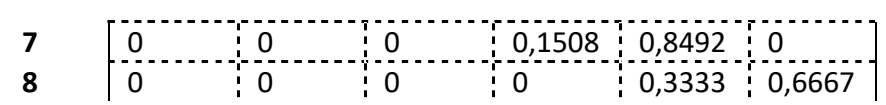

Os dados de vistorias encaminhados da Marechal Rondon trecho 2, do km 158+650 ao km $336+500$ possuem 88 obras vistoriadas. Após a filtragem, os dados de 70 pontes foram usados para a simulação estocástica. As pontes possuem classificações nos oito níveis, no entanto, as transições do IC=2 para IC=1 e IC=3 para IC=2 não possuem dados suficientes para a montagem da MTP, resultando na matriz 6x6 da Equação 12:

\begin{tabular}{|c|c|c|c|c|c|c|}
\hline IC & 3 & 4 & 5 & 6 & 7 & 8 \\
\hline 3 & 1. & 0 & 0 & 0 & 0 & 0 \\
\hline 4 & 0,04 & 0,9600 & 0 & 0 & 0 & 0 \\
\hline 5 & 0 & 0,0074 & 0,9926 & 0 & 0 & 0 \\
\hline 6 & 0 & 0 & 0,0469 & 0,9531 & 0 & 0 \\
\hline 7 & 0 & 0 & 0 & 0,2581 & 0,7419 & 0 \\
\hline 8 & 0 & 0 & 0 & 0 & 0,1667 & 0,8333 \\
\hline
\end{tabular}

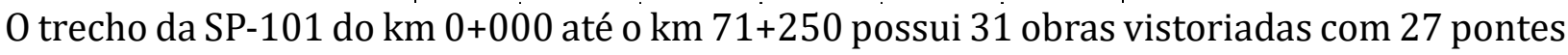
selecionadas para o estudo após a filtragem. Devido ao pequeno número de transições, apesar das pontes apresentarem classificações dos níveis 2 ao 8, a MTP pode ser construída apenas com 4 níveis de avaliação, no intervalo do IC=5 ao IC=8, conforme MTP de tamanho $4 \times 4$ da Equação 13:

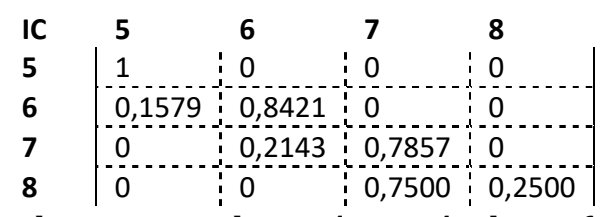

Analisando as MTP das rodovias estaduais é possível verificar a tendência de as pontes permanecerem com a classificação nos IC centrais. Destaca-se no grupo de pontes da Raposo Tavares a alta probabilidade de mudança do melhor nível IC=8 para o nível IC=7 (93,55\%) no curto período de um ano. As probabilidades de mudança dos níveis subsequentes dessa rodovia são menores, no entanto são ainda bastante significativas, em torno de $15 \%$.

Uma tendência levemente diferente da regra observada é verificada na MTP da Marechal Rondon trecho 2, com a alta probabilidade de ocorrer a mudança do IC=7 para o IC=6 com $25,81 \%$ em apenas um ano, probabilidade ainda maior que a tendência de uma ponte do mesmo conjunto mudar do melhor estado IC=8 para o IC=7 (16,67\%).

A partir dos dados da MTP, para a melhor verificação da evolução do processo deteriorativo, foram construídos os gráficos das curvas de deterioração das rodovias paulistas, apresentados na Figura 4:

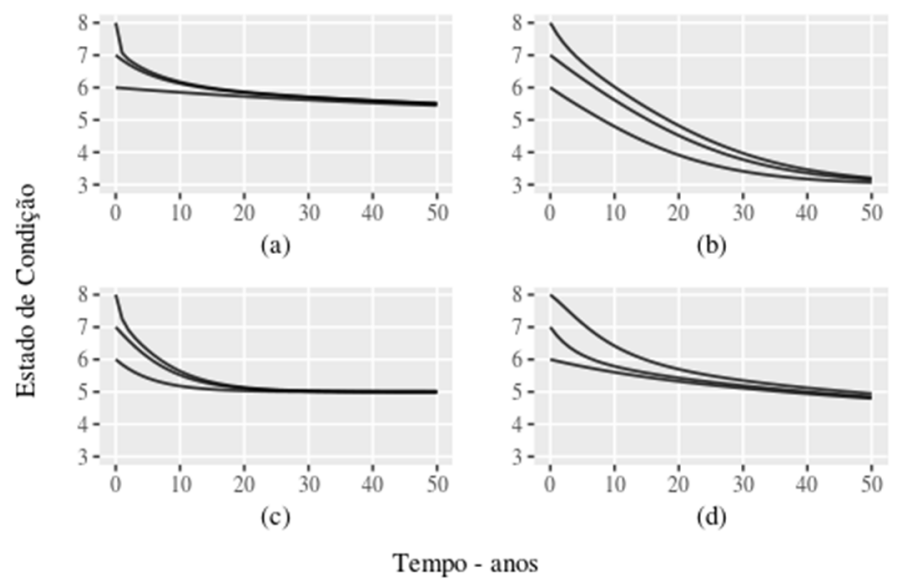

Figura 4: Curvas de deterioração das pontes das rodovias estaduais. (a) Raposo Tavares (b) Rodovia Marechal Rondon trecho 01 (c) Rodovia Tietê-Campinas (d) Rodovia Marechal Rondon trecho 02 
Na Figura 4 é possível verificar que as curvas das pontes da Marechal Rondon - trecho 2 se distanciam bastante do mínimo IC=4, nos primeiros 50 anos. Esse comportamento está associado à distribuição dos valores de IC desse trecho, com forte tendência a permanência nos IC superiores e pequena probabilidade de $4 \%$ de mudança para o IC mais inferior o IC=3. A Tabela 4 apresenta o tempo médio, em anos, das pontes das rodovias passarem de um nível Y para Y-1, de acordo com a MTP estimada até o penúltimo ano da amostragem.

\begin{tabular}{|c|c|c|c|c|c|}
\hline \multirow[b]{2}{*}{ Trecho Rodoviário } & \multicolumn{5}{|c|}{ Período de Transição para um IC inferior (em anos) } \\
\hline & $8 » 7$ & $7 » 6$ & $6 \geqslant 5$ & $5 » 4$ & $4 » 3$ \\
\hline SP270 & 1,0 & 6,6 & 62,9 & - & - \\
\hline SP300 - Trecho 1 & 3,0 & 6,6 & 7,3 & 10,9 & 5,7 \\
\hline SP101 & 1,3 & 4,7 & 6,3 & - & - \\
\hline SP300 - Trecho 2 & 6,0 & 3,9 & 21,3 & 135,1 & 25,0 \\
\hline
\end{tabular}

Com os dados da Tabela 4 é possível verificar que as pontes permanecem pouco tempo com sua classificação no melhor estado. A exceção da SP-300 - trecho 1, as pontes da malha paulista tendem permanecer mais tempo no IC=6. Curiosamente, apesar de não existirem pontes com classificações abaixo do IC=5 na SP-101 observa altas taxas de deterioração de suas obras, com decaimento de 3 níveis de classificação em pouco mais de 12 anos em média. Administrada pela mesma empresa responsável pelo trecho da SP-101, o trecho 2 da SP-300 possui taxas de deterioração inferiores.

Foram construídas novas MTP desconsiderando-se o último ano do ciclo de cada grupo de pontes e os resultados estão apresentados na Tabela 5.

A Tabela 5 mostra a utilização bastante peculiar da escala de classificação do IC por trecho rodoviário. Importante observar que o número previsto de pontes no IC é mais assertivo nos trechos rodoviários com maior número de relatórios de inspeções disponíveis, bem como o desvio é notadamente menor no trecho 2 da SP-300 e na SP-101 com 7 ciclos de dados cada um. Os demais trechos apresentam maior dispersão dos resultados esperados, inclusive quando comparados aos valores esperados das rodovias federais na Tabela 5, atribuída ao limitado período de informações disponíveis e a necessidade da retirada do último ano para a análise.

Tabela 5 - Previsão de número pontes das rodovias estaduais por IC após 1 ciclo

\begin{tabular}{|c|c|c|c|c|c|c|c|c|c|}
\hline \multirow{2}{*}{ Rodovia } & \multirow{2}{*}{ Ano } & \multicolumn{8}{|c|}{ Número de pontes em cada Estado de Condição } \\
\hline & & 1 & 2 & 3 & 4 & 5 & 6 & 7 & 8 \\
\hline \multirow{3}{*}{$\begin{array}{c}\text { SP-300 (Trecho 1) } \\
\text { Empresa Viarondon Concessionária } \\
\text { de Rodovias S/A }\end{array}$} & 2014 (dados) & - & 0 & 2 & 6 & 35 & 69 & 63 & 18 \\
\hline & 2015 (dados) & - & 0 & 3 & 6 & 40 & 72 & 57 & 15 \\
\hline & $\begin{array}{c}\text { Previsão } \\
2015 \\
\end{array}$ & - & 0 & $\begin{array}{c}3 \\
(0,93) \\
\end{array}$ & $\begin{array}{c}8 \\
(1,94) \\
\end{array}$ & $\begin{array}{c}41 \\
(3,32) \\
\end{array}$ & $\begin{array}{c}69 \\
(4,02) \\
\end{array}$ & $\begin{array}{c}60 \\
(3,47) \\
\end{array}$ & $\begin{array}{c}12 \\
(2,00) \\
\end{array}$ \\
\hline \multirow{3}{*}{$\begin{array}{c}\text { SP-300 (Trecho 2) } \\
\text { Empresa Concessionária Rodovias } \\
\text { do Tietê S/A }\end{array}$} & 2015 (dados) & - & - & 1 & 1 & 19 & 31 & 11 & 7 \\
\hline & 2016 (dados) & - & - & 1 & 1 & 21 & 30 & 10 & 7 \\
\hline & $\begin{array}{c}\text { Previsão } \\
2016\end{array}$ & - & - & 1 & 1 & $\begin{array}{c}20 \\
(1,23)\end{array}$ & $\begin{array}{c}33 \\
(1,87)\end{array}$ & $\begin{array}{c}9 \\
(1,75)\end{array}$ & $\begin{array}{c}6 \\
(0,99)\end{array}$ \\
\hline \multirow{3}{*}{$\begin{array}{c}\text { SP-270 } \\
\text { Rodovia Raposo Tavares }\end{array}$} & 2015 (dados) & - & - & - & - & 0 & 22 & 57 & 6 \\
\hline & 2016 (dados) & - & - & - & - & 0 & 29 & 55 & 1 \\
\hline & $\begin{array}{c}\text { Previsão } \\
2016 \\
\end{array}$ & - & - & - & - & $\begin{array}{c}0 \\
(0,59) \\
\end{array}$ & $\begin{array}{c}30 \\
(2,76) \\
\end{array}$ & $\begin{array}{c}54 \\
(2,77) \\
\end{array}$ & $\begin{array}{c}1 \\
(0,60) \\
\end{array}$ \\
\hline \multirow{3}{*}{$\begin{array}{c}\text { SP-101 } \\
\text { Trecho Campinas - Tietê }\end{array}$} & 2015 (dados) & - & - & - & - & 9 & 12 & 6 & 0 \\
\hline & 2016 (dados) & - & - & - & - & 10 & 13 & 4 & 0 \\
\hline & $\begin{array}{c}\text { Previsão } \\
2016\end{array}$ & - & - & - & - & $\begin{array}{c}11 \\
(1,26)\end{array}$ & $\begin{array}{c}11 \\
(1,61)\end{array}$ & $\begin{array}{c}5 \\
(1,00)\end{array}$ & $\begin{array}{c}0 \\
(0,00)\end{array}$ \\
\hline
\end{tabular}




\section{CONCLUSÕES}

Apesar da utilização diferenciada das escalas de qualificação e das diferentes metodologias de inspeção adotadas nas pontes dos trechos rodoviários da pesquisa, todas as MTP resultantes apresentaram altas probabilidades de concentração dos valores de IC em torno da média de cada conjunto, em concordância com estudo de WANG e FOLIENTE (2008) e VARELA (2007) feito com dados de inspeções em pontes norte-americanas. Esses estudos apresentaram resultados que demonstraram a tendência dos avaliadores em não atribuir notas muito ruins ou muito boas para qualquer estrutura, revelando a subjetividade das avaliações nessa área e a tendência comum dos inspetores não apresentarem comportamento muito pessimista ou muito otimista. No entanto, essa concentração em poucos níveis de IC verificada nos dados pode prejudicar a representação fiel do processo de deterioração dessas estruturas, sabidamente de evolução gradual e progressiva.

As altas probabilidades normalmente encontradas nas MTP de transição dos melhores IC para um imediatamente inferior após o breve intervalo de 1 ano não podem ser atribuídas somente ao fenômeno deteriorativo. Metodologias com critérios menos qualitativos e mais técnicos, discriminando com maior detalhamento as condições para a classificação em cada IC poderiam a exemplo de medidas já adotadas pela nova versão da norma ABNT 9452/2016 (ABNT, 2016) podem auxiliar no uso mais eficiente da escala de classificação.

A partir das análises dos resultados, verificou-se a aplicabilidade e razoável acurácia do método de Cadeias de Markov para a previsão do estado futuro de pontes, contribuindo no planejamento de intervenções. A assertividade preditiva dependente diretamente do volume do histórico de dados disponíveis, ou seja, do número de ciclos de inspeção registrados.

Para os casos com avaliação do IC por áreas da estrutura pôde ainda ser verificado que as taxas de deterioração são bastante distintas para diferentes regiões nas pontes. Existe uma tendência de maiores taxas de deterioração para os elementos mais próximos das regiões de impacto direto de tráfego. Portanto, a generalização do estado da ponte em uma única nota global pode ser um empecilho à compreensão integral do processo deteriorativo.

O avanço no conhecimento do comportamento de deterioração dessas estruturas no Brasil demanda a adoção de práticas semelhantes de inspeção ou de uma base comum com o envolvimento dos inspetores. É essencial o registro dos dados em sistema apropriado para utilização em diversas áreas de pesquisa. Muito embora a disponibilidade de dados estrangeiros pudesse socorrer a pesquisa, a particularidade dos agentes deteriorativos, das normas de projetos e de construção, associados à característica do tráfego das vias, limita seu uso ao país de origem, impondo o desenvolvimento de pesquisas nacionais concordes com a realidade brasileira.

\section{AGRADECIMENTOS}

Os autores agradecem ao apoio à pesquisa concedido pelo DNIT, ARTESP, ANTT, Conselho Nacional de Desenvolvimento Científico e Tecnológico (CNPq) e Fundação de Amparo à Pesquisa do Estado de Minas Gerais (FAPEMIG).

\section{REFERENCIAS BIBLIOGRAFICAS}

AASHTO (2013) The Manual for Bridge Evaluation. Washington, USA. American Association of State Highway and Transportation Officials. ISBN: 978-1-56051-557-9.

ABNT. NBR 9542/2016 Vistoria de Pontes e Viadutos de Concreto. Associação Brasileira de Normas Técnicas. Rio de Janeiro, Brasil.

Agrawal, A. K. e A. Kawaguchi (2009) Bridge element deterioration rates: final report, project C-01-51. New York State Department of Transportation, New York, USA. 
Almeida, J. M. M. R. M. O. (2013) Sistema de gestão de pontes com base em custos de ciclo de vida. Tese (doutorado). Faculdade de Engenharia (FEUP), Universidade do Porto, Porto, Portugal.

ARTESP (2007) Resolução ETC-0021. Agência de Transporte do Estado de São Paulo, São Paulo, Brasil.

Bolukbasil, M.; J. Mohammadi e D. Arditi (2004) Estimating the Future Condition of Highway Bridge Components Using National Bridge Inventory Data. Practice periodical on structural design and construction. American Society of Civil Engineers, v.1, p. 16-25. DOI:10.1061/(ASCE)1084-0680(2004)9:1(16).

Campos Neto, C. A. S. (2018) Reflexões sobre investimentos em infraestrutura de transporte no Brasil. Instituto de Pesquisas econômicas aplicadas, IPEA. Disponível em: <http://repositorio.ipea.gov.br/bitstream/11058/7317/1/Radar_n47_reflex\%C3\%B5es.pdf> (acesso em 24/12/2018).

DNIT 010/2004 - PRO Inspeções em pontes e viadutos de concreto armado e protendido - Procedimento. Departamento Nacional de Infraestrutura em Transportes /Instituto de Pesquisas Rodoviárias - IPR, Rio de Janeiro. Disponível em: <http://ipr.dnit.gov.br/normas-e-manuais/normas/procedimento-pro/dnit010_2004_pro.pdf > (acesso em 24/12/2018).

Golabi, K. e R. Shephard (1997) Pontis: A system for maintenance optimization and improvement of US bridge networks. Journal Interfaces, v.1, p.71- 88. DOI:10.1287/inte.27.1.71

Hurt, M. e S. Schrock (2016) Highway Bridge Maintenance, Planning and Scheduling. Elsevier Inc, Kidlington, Oxford, UK. ISBN: 978-0-12-802069-2.

Jiang, Y. (2010) Application and Comparison of Regression and Markov Chain Methods in Bridge Condition Prediction and System Benefit Optimization. Journal of the Transportation Research Forum, v. 49(2), p. 91-110.

Kong, J.S.; J. H. Kim; B. T. Adey e L. Klatter (2010) Trends in development of bridge management systems. Bridge Maintenance, Safety, Management and Life-Cycle Optimization, Londres. ISBN: 9780415877862.

Lima, J. P. (2007) Modelo de decisão para priorização de vias candidatas às atividades de manutenção e reabilitação de pavimentos. Tese (doutorado). Escola de Engenharia de São Carlos, Universidade de São Paulo /USP, São Carlos, São Paulo, Brasil.

Mendes, P. T. C. (2003) Contribuições para um modelo de gestão de pontes de concreto aplicado à rede de rodovias brasileiras. Tese (doutorado), Universidade de São Paulo, São Paulo, Brasil.

Morcous, G. (2000) Case-based reasoning for modeling Bridge Deterioration. Tese (doutorado). Building, Civil and Environmental Engineering, Concordia University, Quebec, Canada.

Morcous, G.; Z. Lounis e M. S. Mirza (2002) Life-cycle assessment of highway bridges. Institute for Research in construction IRC, National Research Council Canada.

Morcous, G. (2006) Performance prediction of Bridge Deck Systems Using Markov Chains. Journal of Performance of Constructed Facilities, v. 20 (2), p. 146-155. DOI:10.1061/(ASCE)0887-3828(2006)20:2(146).

Morcous, G e A. Hatami (2011) Developing Deterioration Models for Nebraska Bridges. University of Nebraska, USA. Disponível em: <https://digitalcommons.unl.edu/matcreports/31/> (acesso em 24/12/2018).

Muñoz, Y. F.; A. Paz, H. Fuente-Mella; J. V. Fariña e G. M. Sales (2016) Estimating Bridge Deterioration by Using Small Data Sets. Universidade de Nevada, Estados Unidos. DOI: 10.1999/1307-6892/43375.

Oliveira, C. B. L e J. M. C. Fonseca (2013) Análise da eficácia do reforço de pontes rodoviárias em concreto armado. 56 Congresso do IBRACON, Natal, Brasil.

Norris, J. R. (1998) Markov Chains. Cambridge Series in Statistical and Probabilistic Mathematics. Cambridge University. ISBN: 978-0521633963.

Setunge, S. e M. S. Hasan (2011) Concrete Bridge Deterioration Prediction using Markov Chain Approach. RMIT University, Melbourne, Austrália. Disponível em: <https://pdfs. semanticscholar.org/46f0/493a796c716768626295bddb0ed1200c1743.pdf>(acesso em 24/12/2018)

Varela, M. L. R. (2007) Uma contribuição para o escalonamento da produção baseado em métodos globalmente distribuídos. Tese (doutorado). Universidade do Minho, Braga, Portugal.

Wang, X e G. Foliente (2008). Identifying bridge structural condition development trends via categorial inspection condition rating with case studies. Structure and Infrasctruture Engineering v. 4, p. 449-466. DOI:10.1080/15732470600924656. 